\title{
Tumor-to-Blood Ratio for Assessment of Somatostatin Receptor Density in Neuroendocrine Tumors Using ${ }^{68}$ Ga-DOTATOC and ${ }^{68}$ Ga-DOTATATE
}

\author{
Ezgi Ilan ${ }^{1,2}$, Irina Velikyan ${ }^{1,3}$, Mattias Sandström ${ }^{1,2}$, Anders Sundin*1,3, and Mark Lubberink*1,2 \\ ${ }^{I}$ Section of Nuclear Medicine and PET, Department of Surgical Sciences, Uppsala University, Uppsala, Sweden; ${ }^{2}$ Medical Physics, \\ Uppsala University Hospital, Uppsala, Sweden; and ${ }^{3}$ PET Centre, Medical Imaging Centre, Uppsala University Hospital, Uppsala, \\ Sweden
}

\begin{abstract}
PET/CT with ${ }^{68} \mathrm{Ga}-\mathrm{DOTA}$-somatostatin analogs has been tested for therapy monitoring in patients with neuroendocrine tumors (NETs). However, SUVs in tumors do not correlate with the net influx rate $\left(\mathrm{K}_{\mathrm{i}}\right)$, as a representation of the somatostatin receptor expression. In this study, tumor-to-blood ratio (TBR) was evaluated as an alternative tool for semiquantitative assessment of ${ }^{68} \mathrm{Ga}$-DOTATOC and ${ }^{68} \mathrm{Ga}$-DOTATATE tumor uptake and as a therapy monitoring tool for patients with NETs. Methods: Twenty-two NET patients underwent a 45-min dynamic PET/CT scan after injection of ${ }^{68} \mathrm{Ga}$-DOTATOC or ${ }^{68} \mathrm{Ga}$-DOTATATE. $\mathrm{K}_{\mathrm{i}}$ was determined using the Patlak method, and TBR was calculated for the 40- to 45-min interval. Results: A linear relation was found between $K_{i}$ and TBR, with a square of Pearson correlation of 0.98 and 0.93 for ${ }^{68} \mathrm{Ga}-$ DOTATOC and ${ }^{68} \mathrm{Ga}-D O T A T A T E$, respectively. Conclusion: $A$ high correlation was found between $\mathrm{K}_{\mathrm{i}}$ and TBR. Hence, TBR reflects somatostatin receptor density more accurately than SUV and is suggested as the preferred metric for semiquantitative assessment of ${ }^{88} \mathrm{Ga}-\mathrm{DOTATOC}$ and ${ }^{68} \mathrm{Ga}-\mathrm{DOTATATE}$ tumor uptake.
\end{abstract}

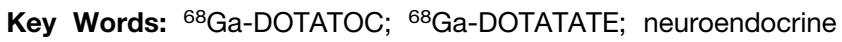
tumors, tumor-to-blood ratio; SUV; net influx rate

J Nucl Med 2020; 61:217-221

DOI: 10.2967/jnumed.119.228072

$\mathbf{N}$ euroendocrine tumors (NETs) are rare neoplasms that arise from endocrine cells distributed throughout the body and have diverse biologic and clinical characteristics (1). The feature of high cellular expression of somatostatin receptors (SSTRs) in NETs enables the use of radiolabeled somatostatin analogs for imaging and therapy. During the past decade, PET using ${ }^{68} \mathrm{Ga}$-labeled somatostatin analogs, such as ${ }^{68} \mathrm{Ga}$-DOTATOC, ${ }^{68} \mathrm{Ga}$-DOTANOC, and ${ }^{68} \mathrm{Ga}$-DOTATATE, has gradually replaced SSTR scintigraphy with ${ }^{111}$ In-DTPA-octreotide (OctreoScan; Mallinckrodt) $(2,3)$ and become the standard method for SSTR imaging of NETs (4).

PET/CT with ${ }^{68} \mathrm{Ga}$-DOTATOC and ${ }^{68} \mathrm{Ga}$-DOTATATE has also been suggested as a tool for evaluation of therapy response in

\footnotetext{
Received Mar. 11, 2019; revision accepted Jun. 18, 2019.

For correspondence or reprints contact: Ezgi Ilan, Uppsala University Hospital, Medical Physics, SE-751 85 Uppsala, Sweden.

E-mail: ezgi.ilan@akademiska.se

${ }^{\star}$ Contributed equally to this work.

Published online Jul. 13, 2019.

COPYRIGHT (C 2020 by the Society of Nuclear Medicine and Molecular Imaging.
}

patients with NETs (5-8). For metabolic tracers such as ${ }^{18} \mathrm{~F}-\mathrm{FDG}$, it can be assumed that the tracer's distribution volume is the whole body since glucose is consumed by all tissues, which means that the SUV can be used as a reasonable measure of metabolism. A challenge with PET/CT using receptor ligands, such as ${ }^{68} \mathrm{Ga}$-DOTATATE and ${ }^{68} \mathrm{Ga}$-DOTATOC, is that the distribution volume instead is confined to those tissues that are, in fact, taking up the tracer, which may affect the SUV quantification. In one study (5), it was found that the changes in tumor SUV between baseline and followup ${ }^{68} \mathrm{Ga}$-DOTATOC PET/CT did not correlate with the therapy outcome of peptide receptor radionuclide therapy. The same finding was also reported for another study (6), although changes in the tumor-to-spleen SUV ratio between baseline and follow-up ${ }^{68} \mathrm{Ga}$ DOTATOC were shown to be more accurate than changes in tumor $\mathrm{SUV}_{\text {max }}$ to evaluate the response to peptide receptor radionuclide therapy. The difficulties of applying static tumor uptake measurements in these 2 therapy monitoring studies may at least partly be explained by the results in a study (7) on tracer kinetics of ${ }^{68} \mathrm{Ga}$ DOTATOC and ${ }^{68} \mathrm{Ga}$-DOTATATE. In that work, net influx rate $\left(\mathrm{K}_{\mathrm{i}}\right)$, assumed to more accurately reflect SSTR density than SUV, was estimated on the basis of dynamic PET imaging, and it was found that SUV saturated (SUV $>20-25$ ) at a static value for high $\mathrm{K}_{\mathrm{i}}$ values $\left(K_{i}>0.2\right)$. Hence, SUV does not appear to reflect SSTR density for tumors with high SSTR expression. The hypothesis of the present work is that saturation in SUV for high $\mathrm{K}_{\mathrm{i}}$ values may be explained by low availability of ${ }^{68} \mathrm{Ga}$-DOTATOC and ${ }^{68} \mathrm{Ga}$-DOTATATE in the blood at some time after administration due to the substantial amounts of SSTR in these patients. Hence, the tumor-to-blood ratio (TBR) may be a better metric than SUV to quantify changes in SSTR expression to assess NET therapy response. The aim of this study was to evaluate the correlation between $\mathrm{K}_{\mathrm{i}}$ and TBR for patients undergoing PET/CT with ${ }^{68} \mathrm{Ga}$-DOTATOC and ${ }^{68} \mathrm{Ga}$-DOTATATE.

\section{MATERIALS AND METHODS}

\section{Patients}

The data in this work were collected from 3 different studies that were approved by the Regional Ethics Review Board in Uppsala. All patients gave written informed consent before inclusion in each study.

The study included 22 patients (11 men and 11 women; mean age, $63 \mathrm{y}$; range, 47-75 y) with histologically confirmed disseminated NETs (10 small-intestinal, 6 pancreatic, 2 rectal, 1 duodenal, 1 lung, and 2 pancreatic neuroendocrine cancers; 4 grade 1 [Ki-67 $\leq 2 \%$ ], 15 grade 2 [Ki-67 $=3-20 \%$ ], 2 grade $3[\mathrm{Ki}-67 \geq 20 \%]$, and 1 without biopsy). The clinical patient data are presented in Table 1 . Some patients were examined with both ${ }^{68} \mathrm{Ga}$-DOTATOC and ${ }^{68} \mathrm{Ga}$-DOTATATE 


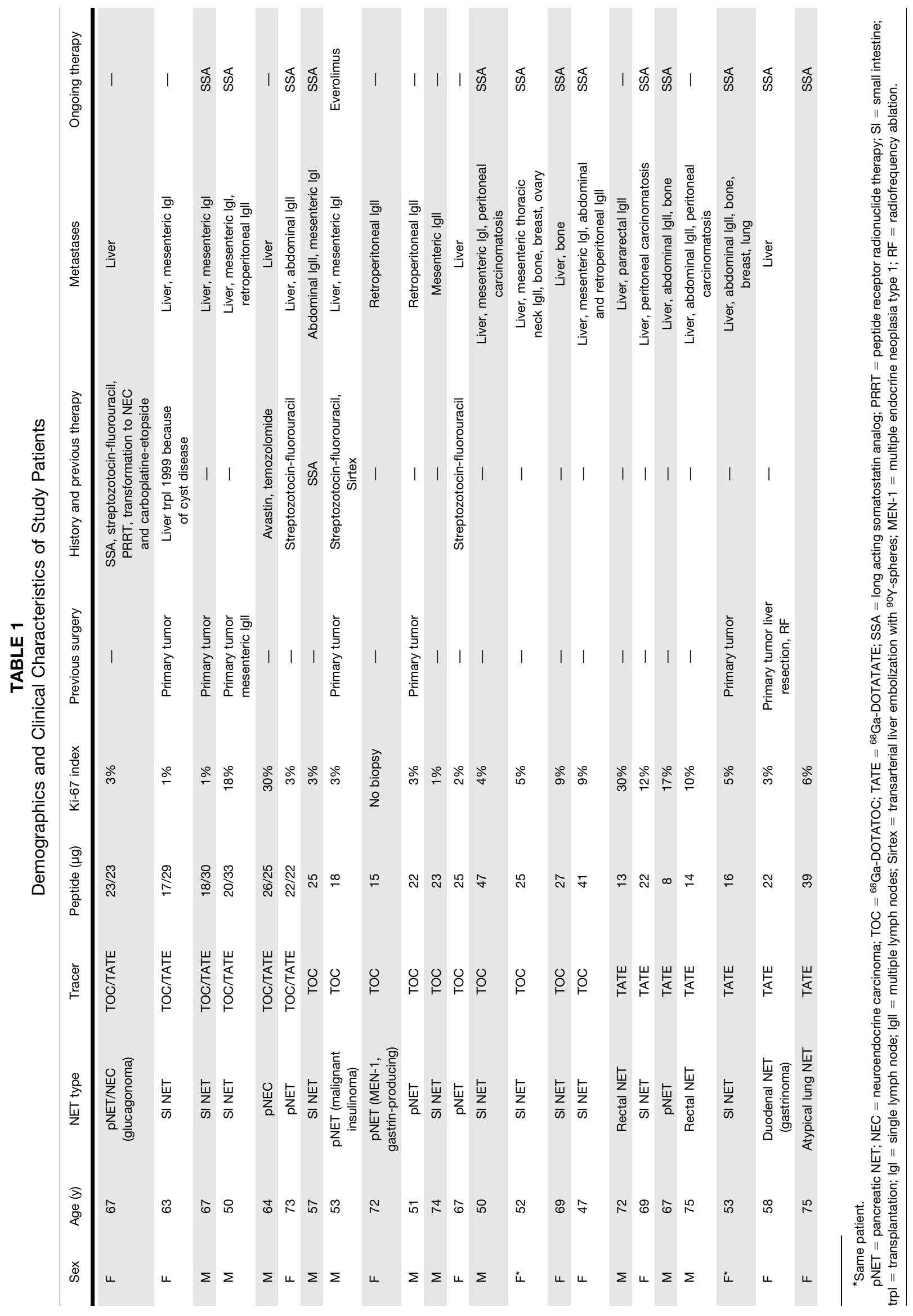


TABLE 2

Reconstruction Settings for the 3 Different Scanners

\begin{tabular}{|c|c|c|c|}
\hline Reconstruction setting & Discovery ST & Discovery IQ & Discovery MI \\
\hline Reconstruction algorithm & OSEM & OSEM with PSF modeling & ToF OSEM with PSF modeling \\
\hline Iterations/subsets & $2 / 28$ & $4 / 12$ & $3 / 16$ \\
\hline Postprocessing filter (mm) & 5 & 4 & 5 \\
\hline Matrix size & $128 \times 128$ & $256 \times 256$ & $256 \times 256$ \\
\hline Pixel size $(\mathrm{mm})$ & $3.91 \times 3.91 \times 3.27$ & $1.95 \times 1.95 \times 3.26$ & $1.95 \times 1.95 \times 2.79$ \\
\hline
\end{tabular}

on consecutive days $(n=6)$, whereas the remainder were examined with either ${ }^{68} \mathrm{Ga}$-DOTATOC or ${ }^{68} \mathrm{Ga}$-DOTATATE. One patient was examined with both ${ }^{68}$ Ga-DOTATOC and ${ }^{68}$ Ga-DOTATATE with 1 y apart. Sixteen patients underwent a ${ }^{68} \mathrm{Ga}$-DOTATOC PET/CT examination after a bolus injection of $131 \pm 47 \mathrm{MBq}, 25 \pm 8 \mu \mathrm{g}$ (range, 62-198 MBq, 15-47 $\mu \mathrm{g}$ ), and 13 patients underwent a ${ }^{68} \mathrm{Ga}$-DOTATATE PET/CT examination after a bolus injection of $107 \pm 31 \mathrm{MBq}, 23 \pm 9 \mu \mathrm{g}$ (range, 76-197 MBq, 8-39 $\mu \mathrm{g})$.

\section{Image Acquisition and Reconstruction}

The patients were examined on a Discovery ST, Discovery IQ or Discovery MI PET/CT scanner (GE Healthcare). They underwent a low-dose CT scan (140 kV; auto mA, 20-80 mA) followed by a 45-min dynamic PET examination of the abdomen. The dynamic PET examination started simultaneously with the intravenous injection of ${ }^{68} \mathrm{Ga}$-DOTATOC or ${ }^{68} \mathrm{Ga}$-DOTATATE and consisted of 22 time frames of increasing duration $(6 \times 10 \mathrm{~s}, 3 \times 20 \mathrm{~s}, 3 \times 60 \mathrm{~s}, 5 \times 180 \mathrm{~s}$, and $5 \times 300 \mathrm{~s})$. All appropriate corrections were applied to the PET data, and the reconstruction settings are specified in Table 2.

\section{Image-Derived Input Functions}

The total radioactivity concentration in the arterial plasma was used as the input function. Volumes of interest were drawn using a $70 \%$ isocontour over the descending thoracic aorta in 10 consecutive image planes in the time frame in which the first passage of the bolus was best visualized (frames 1-10) and then projected onto all time frames in the dynamic examination, generating an arterial time-activity concentration curve (NEDPAS software; VU University Medical Centre, Amsterdam (9)). The image-derived input functions were calculated by multiplying the arterial time-activity concentration curve with a fixed plasma-to-whole-blood ratio of 1.6 based on data from previous work (7) and plasma-to-whole-blood ratio (unpublished data) (mean, 1.6 for both tracers; range, 1.45-1.73). Blood SUV at 40-45 min was determined using the isocontour volume of interest $(70 \%)$ in the descending aorta at the last frame of the dynamic scan.

\section{Kinetic Analysis}

Tumors with a diameter larger than $1 \mathrm{~cm}$ and with high tracer uptake (determined visually) were included for evaluation. Isocontour tumor volumes of interest $(50 \%)$ were drawn in the 20 - to 45 -min (frames 18-22) summation image of the dynamic examination and were projected onto all time frames to generate tumor time-activity concentration curves. $\mathrm{K}_{\mathrm{i}}$ was determined using the Patlak method (10) as previously described (11). SUV and TBR were computed for the last frame of the dynamic scan (i.e., 40-45 min after injection).

\section{Statistical Analysis}

The difference in blood SUV between high $(>0.2)$ and low $(<0.2)$ $\mathrm{K}_{\mathrm{i}}$ values was determined using a Mann-Whitney test, with the significance level set to a $P$ value of less than 0.05 (Prism, version 6.07; GraphPad Software, Inc.). In this test, only 1 tumor per patient was included (the tumor with the highest $\mathrm{K}_{\mathrm{i}}$ ) because some patients had several tumors whereas others had only one, and inclusion of all tumors would bias the results toward patients with more tumors.

The relation between $\mathrm{K}_{\mathrm{i}}$ and TBR was evaluated using linear regression and Pearson correlation and compared with the relation between $\mathrm{K}_{\mathrm{i}}$ and SUV. In this test, all tumors were included.

$\mathrm{K}_{\mathrm{i}}, \mathrm{SUV}_{\text {tumor }}$ and TBR were also compared between ${ }^{68} \mathrm{Ga}$-DOTATOC and ${ }^{68} \mathrm{Ga}$-DOTATATE using Deming regression, Pearson correlation, and Wilcoxon matched-pairs tests (significance level set to $P<0.05$ ).

\section{RESULTS}

In total, 71 tumors were included in the study: 38 for ${ }^{68} \mathrm{Ga}$ DOTATOC (6 patients with 1 tumor, 3 with 2 tumors, 3 with 3 tumors,

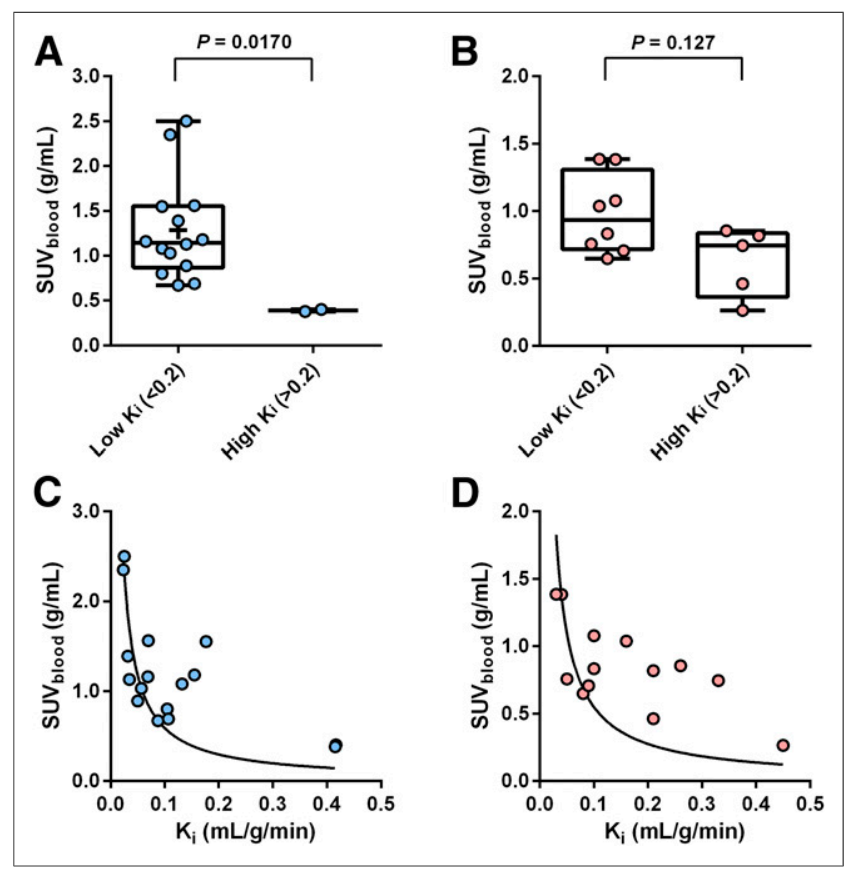

FIGURE 1. (A and B) Box plots of SUV in blood at 40-45 min after injection for ${ }^{68} \mathrm{Ga}$-DOTATOC (A) and ${ }^{68} \mathrm{Ga}$-DOTATATE (B) for high and low $\mathrm{K}_{\mathrm{i}}$ values. One tumor per patient is included in plots. Boxes are median and interquartile range, and whiskers are full range of data. Significant differences $(P<0.05)$ were found in $\mathrm{SUV}_{\text {blood }}$ between high and low $\mathrm{K}_{\mathrm{i}}$ for ${ }^{68} \mathrm{Ga}-$ DOTATOC, however, not for ${ }^{68} \mathrm{Ga}-\mathrm{DOTATATE}(P>0.05)$. (C and D) Relation between $\mathrm{K}_{\mathrm{i}}$ and SUV in blood for ${ }^{8} \mathrm{Ga}$-DOTATOC (C) and ${ }^{68} \mathrm{Ga}$-DOTATATE (D). Solid line represents exponential fit $(y=a / x)$ for visual illustration. 


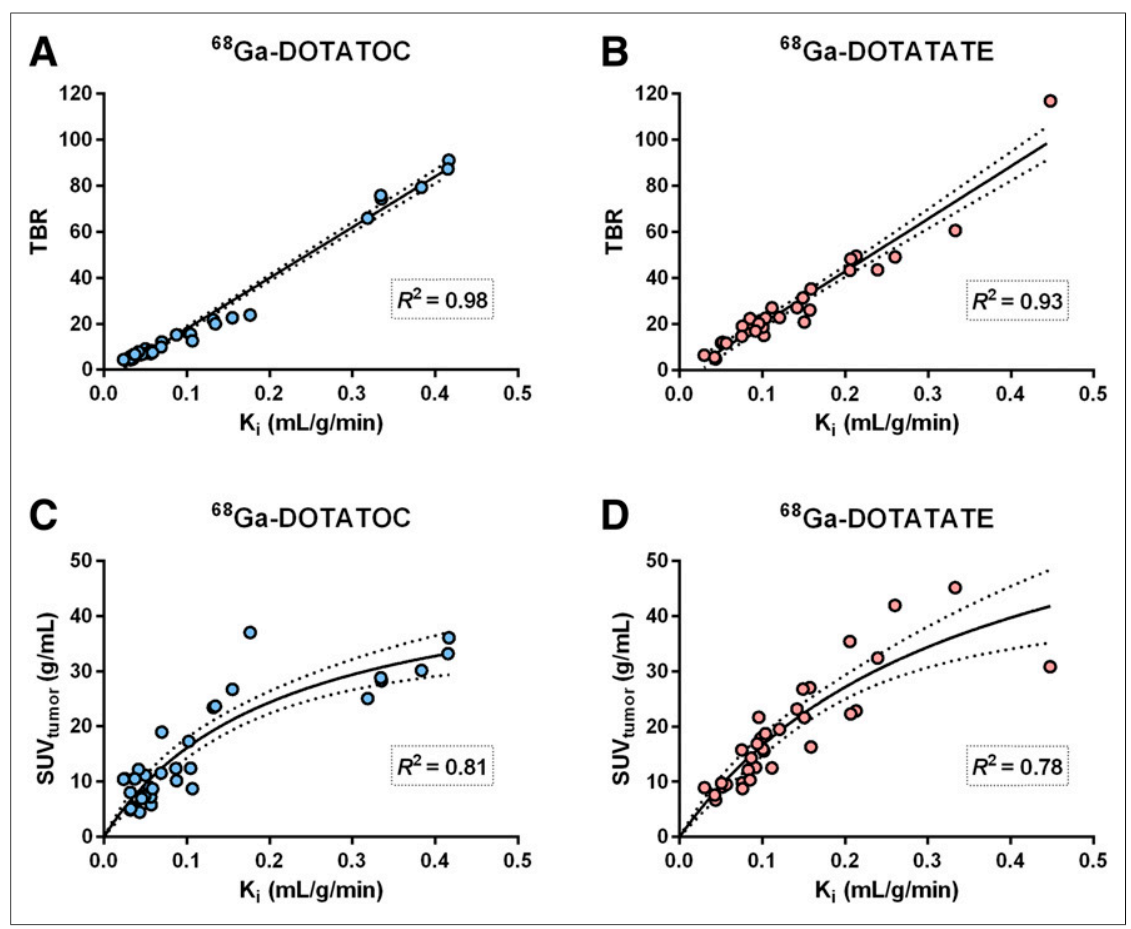

FIGURE 2. Correlation between $\mathrm{K}_{\mathrm{i}}$ and TBR for ${ }^{68} \mathrm{Ga}-\mathrm{DOTATOC}(\mathrm{A})$ and ${ }^{68} \mathrm{Ga}$-DOTATATE (B) and between $\mathrm{K}_{\mathrm{i}}$ and SUV in tumors for ${ }^{68} \mathrm{Ga}$-DOTATOC (C) and ${ }^{8}{ }^{8} \mathrm{Ga}$-DOTATATE (D). Solid lines represent linear regression fits ( $A$ and $B$ ) and fits to hyperbolic line $(C$ and $D)$, and dashed lines are $95 \%$ confidence band of these fits.

3 with 4 tumors, and 1 with 5 tumors) and 33 for ${ }^{68} \mathrm{Ga}$-DOTATATE (4 patients with 1 tumor, 4 with 2 tumors, 2 with 3 tumors, 1 with 4 tumors, 1 with 5 tumors, and 1 with 6 tumors). For ${ }^{68}$ Ga-DOTATOC, SUV in aortal blood at $45 \mathrm{~min}$ after injection was significantly lower in patients with high $\mathrm{K}_{\mathrm{i}}$ values than in those with low $\mathrm{K}_{\mathrm{i}}$ values $(P=0.017$, Mann-Whitney test; Fig. 1A). The difference was smaller for ${ }^{68} \mathrm{Ga}$-DOTATATE $(P=0.127$, Mann-Whitney test; Fig. 1B). The relation between SUV in blood and $\mathrm{K}_{\mathrm{i}}$ is presented in Figures $1 \mathrm{C}$ and $1 \mathrm{D}$ for ${ }^{68} \mathrm{Ga}$-DOTATOC and ${ }^{68} \mathrm{Ga}$-DOTATATE, respectively.

A linear relation was found between $\mathrm{K}_{\mathrm{i}}$ and TBR (all tumors included), with a square of Pearson correlation of 0.98 and 0.93 for ${ }^{68} \mathrm{Ga}-$ DOTATOC and ${ }^{68} \mathrm{Ga}$-DOTATATE, respectively (Fig. 2). $\mathrm{K}_{\mathrm{i}}$ was
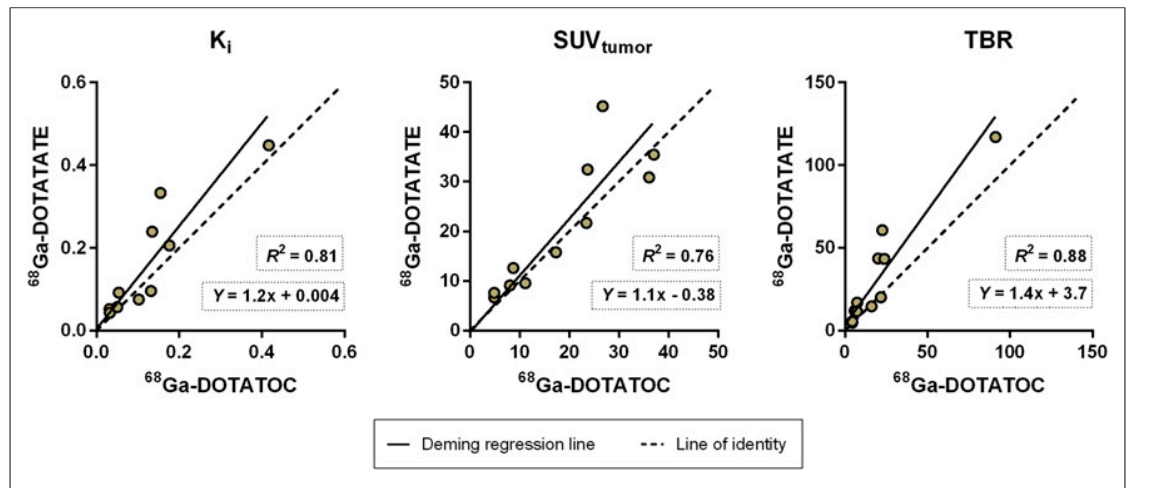

FIGURE 3. Comparison of $\mathrm{K}_{\mathrm{i}}$, SUV $\mathrm{V}_{\text {tumor }}$, and TBR between ${ }^{68} \mathrm{Ga}$-DOTATOC and ${ }^{68} \mathrm{Ga}$-DOTATATE.

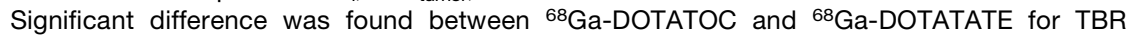
$\left(P=0.019\right.$, Wilcoxon matched-pairs test) but not for $\mathrm{K}_{\mathrm{i}}$ or $\operatorname{SUV}_{\text {tumor }}(P=0.083$ and 0.413 , respectively). compared with SUV for the same tumors, and the relation is illustrated in Figures 2C and 2D. The square of Pearson correlation between $\mathrm{K}_{\mathrm{i}}$ and tumor SUV using a hyperbolic fit was 0.81 and 0.78 for ${ }^{68} \mathrm{Ga}$-DOTATOC and ${ }^{68} \mathrm{Ga}-$ DOTATATE, respectively. Tumor SUV, blood SUV, TBR, and $\mathrm{K}_{\mathrm{i}}$ for each patient are also presented in Supplemental Tables 1 and 2 for both tracers (supplemental materials are available at http://jnm.snmjournals.org).

A significant difference in TBR was found between ${ }^{68} \mathrm{Ga}$-DOTATOC and ${ }^{68} \mathrm{Ga}$ - DOTATATE $(P=0.019$, Wilcoxon matched-pairs test). However, for $\mathrm{SUV}_{\text {tumor }}$ and $\mathrm{K}_{\mathrm{i}}$, there were no significant differences ( $\mathrm{SUV}_{\text {tumor }} P=0.413$; $\mathrm{K}_{\mathrm{i}}, P=0.083$; Wilcoxon matched-pairs test). A linear relation between ${ }^{68} \mathrm{Ga}$-DOTATOC and ${ }^{68} \mathrm{Ga}$-DOTATATE was found for $\mathrm{K}_{\mathrm{i}}, \mathrm{SUV}_{\text {tumor }}$ and TBR, with a square of Pearson correlation of $0.81,0.76$, and 0.88 , respectively (Fig. 3). The slopes of the Deming regression line were $1.2,1.1$, and 1.4 for $\mathrm{K}_{\mathrm{i}}, \mathrm{SUV}_{\mathrm{tumor}}$, and TBR, respectively.

\section{DISCUSSION}

Early prediction of treatment response is essential to guide tumor therapy and avoid unnecessary side effects and costs from ineffective treatments. SUV has been proposed as a measure of SSTR density in NETs, but changes of the tumor SUV in ${ }^{68} \mathrm{Ga}$-DOTATOC PET/CT during peptide receptor radionuclide therapy have not been found to reliably correlate with the treatment outcome $(5,6,8) . \mathrm{K}_{\mathrm{i}}$ is likely to reflect the tumor SSTR density more adequately than SUV. In a previous study (7) comparing ${ }^{68} \mathrm{Ga}$-DOTATOC and ${ }^{68} \mathrm{Ga}$-DOTATATE, it was shown that $\mathrm{K}_{\mathrm{i}}$ and SUV did not correlate linearly for NETs, especially at high SUVs (>20-25). The present work partly used data from the same subjects, and as seen in Figures $2 \mathrm{C}$ and 2D, the addition of more subjects did not alter this conclusion.

This study suggests that the nonlinear relation between $K_{i}$ and SUV for high $\mathrm{K}_{\mathrm{i}}$ values can be attributed to faster blood clearance in patients with a high tumor receptor expression. For ${ }^{68} \mathrm{Ga}$-DOTATOC, SUV in blood at $45 \mathrm{~min}$ after injection was significantly lower in patients with high $\mathrm{K}_{\mathrm{i}}$ values than in those with low $\mathrm{K}_{\mathrm{i}}$ values (Fig. 1A). For ${ }^{68} \mathrm{Ga}$-DOTATATE, this difference was not significant (Fig. 1B). The low blood SUV in patients with high $\mathrm{K}_{\mathrm{i}}$ values may be overestimated because of spill-in from surrounding tissues and a positive bias in lowactivity areas as commonly seen in PET, whereas the high blood SUV may be underestimated because of the partial-volume effect. Taking these factors into consideration, the difference in blood SUV between the 2 groups would increase even further.

Figures 2A and 2B clearly illustrate that, contrary to the nonlinear relation between $\mathrm{K}_{\mathrm{i}}$ and $\mathrm{SUV}$, there is a linear relation between $\mathrm{K}_{\mathrm{i}}$ and TBR and that the nonlinear correlation 
between $\mathrm{K}_{\mathrm{i}}$ and SUV can be attributed to low availability of tracer in blood. Since plasma concentrations during the course of the scan are implicitly considered when estimating $K_{i}$, differences in plasma concentration of the tracer do not affect accuracy. However, since the low blood activity concentrations will limit the absolute amount of tracer available for uptake in tissue, SUV will be affected by low plasma concentrations and will not always follow $\mathrm{K}_{\mathrm{i}}$. Most probably, the total amount of SSTR in some patients is so large that nearly all peptide is cleared from the plasma during the initial part of the examination, leading to the apparent saturation of tumor SUVs. The clearance rate for the individual patient also depends on, for example, kidney function, uptake in kidneys, and spleen. However, the amount of tracer in blood is probably one of the most important factors; moreover, the amount of tracer in blood is a factor that may be influenced by how much peptide is administered, whereas the SSTR expression in each tumor, the combined total SSTR expression, and the patient's renal function cannot be affected.

The activity concentrations in blood were determined by delineating the aorta using a $70 \%$ isocontour volume of interest. In this case, the underestimation of the activity concentration in the aorta is theoretically $6 \%-7 \%$ when assuming an aorta diameter of $2.5 \mathrm{~cm}$ and a spatial resolution of $5 \mathrm{~mm}$. The results in this paper were not corrected for this underestimation. However, since this underestimation will affect $\mathrm{K}_{\mathrm{i}}$ and TBR equally, the conclusions of the study would not change.

The patients in this study were from 3 different NET studies and thus underwent examinations on different scanners with varied reconstruction settings. As known, reconstruction parameters affect $\mathrm{K}_{\mathrm{i}}$ and SUV (12). However, since the reconstruction will affect $K_{i}$ and SUV similarly, the variations in reconstruction settings between scanners will not affect the conclusions of the present work. For example, the partial-volume effect will similarly affect $\mathrm{K}_{\mathrm{i}}$ and SUV and the results will consequently be the same regardless of whether the reconstruction includes correction for partial-volume effect or not.

\section{CONCLUSION}

A linear relation with a high correlation was found between $K_{i}$ and TBR for both ${ }^{68} \mathrm{Ga}$-DOTATOC and ${ }^{68} \mathrm{Ga}$ DOTATATE. Hence, TBR reflects SSTR density better than SUV and would be the preferred measurement tool for semiquantitative assessment of ${ }^{68} \mathrm{Ga}$-DOTATOC and ${ }^{68} \mathrm{Ga}$-DOTATATE tumor uptake and as a means for NET therapy monitoring.

\section{DISCLOSURE}

No potential conflict of interest relevant to this article was reported.

\section{ACKNOWLEDGMENT}

We thank the staff of the PET Centre for their assistance with the PET/CT examinations.

KEY POINTS

QUESTION: The purpose of this study was to evaluate the use of TBR as an alternative tool for semiquantitative assessment of ${ }^{68} \mathrm{Ga}$ DOTATOC and ${ }^{68} \mathrm{Ga}-D O T A T A T E$ tumor uptake and as a therapy monitoring tool for patients with NETs by evaluating the relation

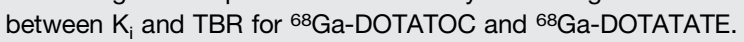

PERTINENT FINDINGS: For both ${ }^{68 \mathrm{Ga}-D O T A T O C}$ and ${ }^{68} \mathrm{Ga}-$ DOTATATE, a linear relation with a high correlation was found between $\mathrm{K}_{\mathrm{i}}$ and TBR. Hence, TBR can be used as a tool for semiquantitative assessment of ${ }^{68} \mathrm{Ga}-\mathrm{DOTATOC}$ and ${ }^{68} \mathrm{Ga}-\mathrm{DOTATATE}$ tumor uptake and as a means for NET therapy monitoring.

IMPLICATIONS FOR PATIENT CARE: The finding offers a new tool for assessing tumor uptake of ${ }^{88} \mathrm{Ga}-D O T A T O C$ and ${ }^{68} \mathrm{Ga}-$ DOTATATE and a new therapy monitoring tool for patients with NETs.

\section{REFERENCES}

1. Sundin A, Rockall A. Therapeutic monitoring of gastroenteropancreatic neuroendocrine tumors: the challenges ahead. Neuroendocrinology. 2012;96:261-271.

2. Krenning EP, Kwekkeboom DJ, Bakker WH, et al. Somatostatin receptor scintigraphy with [ $\left.{ }^{111} \mathrm{In}-\mathrm{DTPA}-\mathrm{D}-\mathrm{Phe} 1\right]$ - and $\left[{ }^{123} \mathrm{I}-\mathrm{Tyr} 3\right]$-octreotide: the Rotterdam experience with more than 1000 patients. Eur J Nucl Med. 1993;20:716-731.

3. Lamberts SW, Bakker WH, Reubi JC, Krenning EP. Somatostatin-receptor imaging in the localization of endocrine tumors. N Engl J Med. 1990;323:1246-1249.

4. Sundin A, Arnold R, Baudin E, et al. ENETS consensus guidelines for the standards of care in neuroendocrine tumors: radiological, nuclear medicine \& hybrid imaging. Neuroendocrinology. 2017;105:212-244.

5. Gabriel M, Oberauer A, Dobrozemsky G, et al. ${ }^{68}$ Ga-DOTA-Tyr3-octreotide PET for assessing response to somatostatin-receptor-mediated radionuclide therapy. J Nucl Med. 2009;50:1427-1434.

6. Haug AR, Auernhammer CJ, Wangler B, et al. ${ }^{68}$ Ga-DOTATATE PET/CT for the early prediction of response to somatostatin receptor-mediated radionuclide therapy in patients with well-differentiated neuroendocrine tumors. J Nucl Med. 2010;51:1349-1356.

7. Velikyan I, Sundin A, Sorensen J, et al. Quantitative and qualitative intrapatient comparison of ${ }^{68} \mathrm{Ga}$-DOTATOC and ${ }^{68} \mathrm{Ga}$-DOTATATE: net uptake rate for accurate quantification. $J$ Nucl Med. 2014;55:204-210.

8. Kratochwil C, Stefanova M, Mavriopoulou E, et al. SUV of $\left[{ }^{68} \mathrm{Ga}\right]$ DOTATOC$\mathrm{PET} / \mathrm{CT}$ predicts response probability of PRRT in neuroendocrine tumors. Mol Imaging Biol. 2015;17:313-318.

9. Boellaard R, Oyen WJ, Hoekstra CJ, et al. The Netherlands protocol for standardisation and quantification of FDG whole body PET studies in multi-centre trials. Eur J Nucl Med Mol Imaging. 2008;35:2320-2333.

10. Patlak CS, Blasberg RG. Graphical evaluation of blood-to-brain transfer constants from multiple-time uptake data: generalizations. J Cereb Blood Flow Metab. 1985;5:584-590.

11. Ilan E, Sandstrom M, Velikyan I, Sundin A, Eriksson B, Lubberink M. Parametric net influx rate images of ${ }^{68} \mathrm{Ga}$-DOTATOC and ${ }^{68} \mathrm{Ga}$-DOTATATE: quantitative accuracy and improved image contrast. J Nucl Med. 2017;58:744-749.

12. Boellaard R. Need for standardization of ${ }^{18}$ F-FDG PET/CT for treatment response assessments. J Nucl Med. 2011;52(suppl 2):93S-100S. 